\title{
NEW SHARP ESTIMATES OF THE GENERALIZED EULER-MASCHERONI CONSTANT
}

\author{
VASILE BERINDE AND CRISTINEL MORTICI
}

Abstract. The aim of this paper is to establish new sequences which converge towards the EulerMascheroni constant. Our results solve some open problems posed by Berinde [A new generalization of Euler's constant Creat. Math. Inform. 18 (2009) no. 2 123-128] and extend some results of DeTemple, [A quicker convergence to Euler's constant Amer. Math. Monthly 100 (1993) 468-470] and Sîntămărian [A generalization of Euler's constant, Numer. Algorithms 46 (2007), 141-151].

Mathematics subject classification (2010): 33B15, 41A10, 42A16.

Keywords and phrases: Euler-Mascheroni constant, inequalities, approximations.

\section{REFERENCES}

[1] H. Alzer, Inequalities for the gamma and polygamma functions, Abh. Math. Sem. Univ. Hamb. 68 (1998), 363-372.

[2] V. BERINDE, A new generalization of Euler's constant, Creat. Math. Inform. 18, 2 (2009), 123-128.

[3] D. W. De Temple, A quicker convergence to Euler's constant, Amer. Math. Monthly 100, 5 (1993), 468-470.

[4] E. A. Karatsuba, On the computation of the Euler constant $\gamma$, Computational methods from rational approximation theory (Wilrijk, 1999), Numer. Algorithms 24, 1-2 (2000), 83-97.

[5] K. KNOPP, Theory and Applications of Infinite Series, Blackie, London, 453 (1951).

[6] C. Mortici, Best estimates of the generalized Stirling formula, Appl. Math. Comput. 215, 11 (2010), 4044-4048.

[7] C. MoRTiCI, Product approximations via asymptotic integration, Amer. Math. Monthly 117, 5 (2010), $434-441$.

[8] C. MORTICI, An ultimate extremely accurate formula for approximation of the factorial function, Arch. Math. (Basel) 93, 1 (2009), 37-45.

[9] C. Mortici, New approximations of the gamma function in terms of the digamma function, Appl. Math. Lett. 23, 1 (2010), 97-100.

[10] C. Mortici, Complete monotonic functions associated with gamma function and applications, Carpathian J. Math. 25, 2 (2009), 186-191.

[11] S.-L. QIU, M. Vuorinen, Some properties of the gamma and psi functions with applications, Math. Comp. 74, 250 (2005), 723-742.

[12] A. SînTĂMĂRIAN, A generalization of Euler's constant, Numer. Algorithms 46, 2 (2007), 141-151.

[13] L. Tóth, Problem E 3432, Amer. Math. Monthly 98, 3 (1991), 264.

[14] R. M. YounG, Euler's constant, Math. Gaz. 75, 472 (1991), 187-190. 